\title{
Synthesis of silica nanosphere from homogeneous and heterogeneous systems
}

\author{
N VENKATATHRI \\ Department of Chemistry, Anna University, Chennai 600 025, India
}

MS received 11 September 2006; revised 24 September 2007

\begin{abstract}
Silica nanosphere was synthesized using homogeneous and heterogeneous systems, respectively. In homogeneous system, silica spheres were synthesized without cetyltrimethylammonium bromide (CTABr), which gave bimodal particle size and lower yield (77\%). To improve the yield, CTABr was added and found that the yield was very high $(100 \%)$. The particle was in $\mathrm{nm}$ range, but the particle sizes are bimodal. To avoid it, reaction in heterogeneous system using $\mathrm{CTABr}$ was carried out. Nanosized silica sphere with uniform size (yield, 94\%) was observed. Homogeneous system contains a mixture of ethanol, water, aqueous ammonia and tetraethylorthosilicate (TEOS). In the case of heterogeneous system, only ethanol was absent.
\end{abstract}

Keywords. Silica nanosphere; homogeneous; heterogeneous.

\section{Introduction}

Amorphous silica nanoparticles are used in many applications including ceramics, catalysis, pharmaceutical, electronic packaging, photonic and chemical-mechanical polishing (Iler 1979; Sacks and Tseng 1984; Masuda et al 1990; Yamashita et al 1992; Taira and Yamaki 1995). In particular, special attention has recently been paid to methods for controlling the size and distribution of nanospheres, because they exhibit peculiar and desirable properties in the wafer polishing process. Monodisperse silica nanospheres were first synthesized by Stober et al (1968) using sol-gel method which induces high purity in the resulting particles. Bogush and Zukoski (1988) reported the influence of reaction parameters such as ammonia and water contents on the particle size and distribution.

Recently, silica nanospheres have been considered as effective candidates for chemical-mechanical polishing materials. So several investigators have reported ways to control particle size by using reactor type and varying the concentrations of ammonia, water and alcohol solvent (Sadasivan et al 1998; Kim et al 2000; Kim and Kim 2002; Park et al 2002). They also examined the influence of reaction methods (such as semi-batch reaction and batch reaction) on particle size and its distribution. A relatively slow rate of hydrolysis of TEOS occurred during the semi-batch process, which resulted in larger silica particles and a narrower size distribution (Kim and Kim 2002).

The mechanism of silica nanospheres formation was generally suggested to be via hydrolysis of silica source

(venkatathrin@yahoo.com) to form the singly hydrolyzed monomer. Subsequently, this intermediate reaction product condensed and eventually formed nanosphere.

\section{Experimental}

\subsection{Synthesis by homogeneous system without CTABr}

Tetraethylorthosilicate, $1.06 \mathrm{ml}(98 \%$, Aldrich, USA) was mixed with $5 \mathrm{ml}$ of ethanol (98\%, Aldrich, USA). To this mixture, $0.215 \mathrm{ml}$ of aqueous ammonia (26-30\%, Aldrich, USA) was added. Then $1.08 \mathrm{ml}$ of distilled water was added. Finally $5 \mathrm{ml}$ of ethanol was added. The total mixture was stirred continuously for $2 \mathrm{~h}$. The product was centrifuged and washed with distilled water several times.

\subsection{Synthesis by homogeneous system with CTABr}

Cetyltrimethylammonium bromide, $2 \cdot 5 \mathrm{~g}$ (surfactant, Aldrich, USA) was dissolved in $50 \mathrm{ml}$ of deionized water, and $13.2 \mathrm{ml}$ of aqueous ammonia and $60 \mathrm{ml}$ of absolute ethanol were added to the surfactant solution. The solution was stirred for $15 \mathrm{~min}(250 \mathrm{rpm})$ and $4.6 \mathrm{ml}$ of TEOS was added once resulting in a gel with the following molar composition: 1 TEOS: $0 \cdot 3 \mathrm{C}_{16}$ TMABr: $11 \mathrm{NH}_{3}$ : $144 \mathrm{H}_{2} \mathrm{O}$ : $58 \mathrm{EtOH}$. After stirring for $2 \mathrm{~h}$, the white precipitate was filtered and washed with distilled water several times.

\subsection{Synthesis by heterogeneous system with $\mathrm{CTABr}$}

Cetyltrimethylammonium bromide $(2 \cdot 405 \mathrm{~g})$ was dissolved in $120 \mathrm{ml}$ of distilled water and $9.5 \mathrm{ml}$ of aqueous 

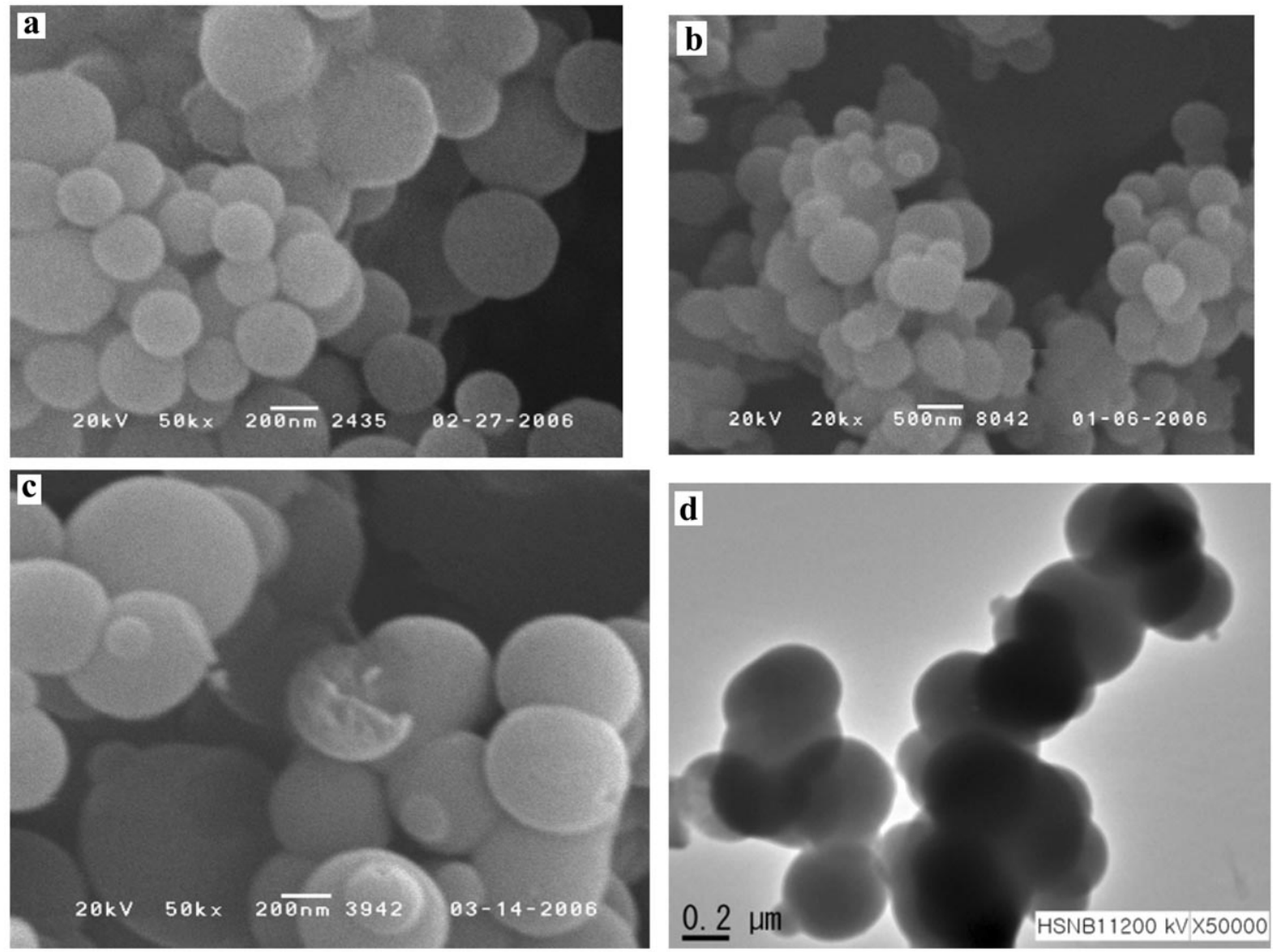

Figure 1. SEM picture of silica nanosphere synthesized from a. homogeneous system without CTABr, b. homogeneous system with $\mathrm{CTABr}$, c. heterogeneous system with $\mathrm{CTABr}$, and $\mathbf{d}$. TEM picture of heterogeneous system sample with CTABr

Table 1. Molar gel compositions of various processes to synthesize silica nanosphere.

\begin{tabular}{llcrl}
\hline S1. no. & \multicolumn{1}{c}{ Gel composition } & Growth period & Yield (\%) & Particle size \\
\hline 1. & 0.5 TEOS: $0.7 \mathrm{NH}_{3}: 6 \mathrm{H}_{2} \mathrm{O}: 85 \mathrm{EtOH}$ & $2 \mathrm{~h}$ & 77 & 100 and $300 \mathrm{~nm}$ \\
2. & 1 TEOS: $0 \cdot 3 \mathrm{C}_{16} \mathrm{TMABr}: 11 \mathrm{NH}_{3}: 144 \mathrm{H}_{2} \mathrm{O}: 58 \mathrm{EtOH}$ & $2 \mathrm{~h}$ & 100 & $200-500 \mathrm{~nm}$ \\
3. & 1 TEOS: $0.152 \mathrm{CTABr}: 2 \cdot 8 \mathrm{NH}_{3}: 141 \cdot 2 \mathrm{H}_{2} \mathrm{O}$ & $1 \mathrm{~h}$ & 94 & $200 \mathrm{~nm}$ \\
\hline
\end{tabular}

Conditions: temperature $=25^{\circ} \mathrm{C}$; pressure $=$ ambient.

ammonia was added to the solution. While stirring, $10 \mathrm{ml}$ of tetraethylorthosilicate was added slowly to the surfactant solution over a period of 15 min resulting in a gel with the following molar composition: 1 TEOS: $0 \cdot 152$ CTABr: $2.8 \mathrm{NH}_{3}: 141.2 \mathrm{H}_{2} \mathrm{O}$. The mixture was stirred for $1 \mathrm{~h}$, then the white precipitate was filtered and washed with $100 \mathrm{ml}$ of deionized water.

After drying at $363 \mathrm{~K}$ for $12 \mathrm{~h}$, the samples were heated to $823 \mathrm{~K}$ (rate, $1 \mathrm{~K} / \mathrm{min}$ ) in air and kept at this temperature for $8 \mathrm{~h}$ to remove the template.

The particle size and shape were analysed by a Topcon, SM-300 scanning electron microscope. The copper disc was pasted with carbon tape and the sample was dispersed over the tape. The disc was coated with gold in ionization chamber before microscopic analysis.

\section{Results and discussion}

The SEM pictures of the sample synthesized (table 1) from homogeneous and heterogeneous systems are given in figure 1. Initially, silica nanosphere was synthesized in homogeneous system which involves TEOS, ethanol, aqueous ammonia and water, for $2 \mathrm{~h}$. We found the particle size to be bimodal (100 and $300 \mathrm{~nm}$ ). The yield also was less (77\%). To avoid this, a calculated amount of cetyltri- 
methylammonium bromide was added, which increased the yield considerably $(100 \%)$. Yield was calculated based on the theoretical yield from the total silica input. Here the particle sizes are not even $200-400 \mathrm{~nm}$. To avoid the multiple size of particles, we changed the system into heterogeneous, where the reaction was carried out in absence of ethanol. Water and TEOS did not mix with each other. To avoid this, cetyltrimethylammonium bromide surfactant was added. Now in lesser time $(1 \mathrm{~h})$ of particle growth, good yield and even particle size $(200 \mathrm{~nm})$ occurred. The even particle size of heterogeneous system is also supported by TEM (figure 1d). No any hollow sphere nature in its TEM picture was observed, even though it shows a high surface area $\left(>1000 \mathrm{~m}^{2} / \mathrm{g}\right)$ after calcination.

\section{Conclusions}

Silica nanosphere with uniform size and higher yield was synthesized by modified procedure, which employs $\mathrm{CTABr}$ in heterogeneous system. Earlier, in our attempt with homogeneous system, with and without cetyltrimethylammonium bromide, we obtained less yield with multiple particle sizes.

\section{Acknowledgement}

The author thanks the Council of Scientific and Industrial Research, New Delhi, for a fellowship.

\section{References}

Bogush G H and Zukoski C F 1988 J. Non-Cryst. Solids 104 95

Iler R K 1979 The chemistry of silica (New York: Wiley)

Kim K D and Kim H T 2002 J. Sol-Gel Sci. Technol. 25183

Kim K S, Kim S S, Kim S K, Kim J K and Kim W S 2000 Hwahak Konghak 38817

Masuda R, Takahashi W and Ishii M 1990 J. Non-Cryst. Solids 121389

Park S K, Kim K D and Kim H T 2002 Colloids Surfaces A: Physicochem. Eng. Aspects 1977

Sacks M D and Tseng T Y 1984 J. Am. Ceram. Soc. 67526

Sadasivan S, Rasmussen D H, Chen F P and Kannabiran R K 1998 Colloids Surfaces A: Physicochem. Eng. Aspects 13245

Stober W, Fink A and Bohn E 1968 J. Colloid Interface Sci. 26 62

Taira M and Yamaki M 1995 J. Mater. Sci. Mater. Med. 6197

Yamashita D M, Mori H and Maekawa T 1992 J. Ceram. Soc. Jpn 1001444 